\title{
Ultrafast laser-matter interaction with nanostructured targets
}

\author{
Robin S. Marjoribanks ${ }^{\mathrm{a}}$, L. Lecherbourg ${ }^{\mathrm{a}}$, J.E. Sipe ${ }^{\mathrm{a}}$, G. Kulcsár ${ }^{\mathrm{a}}$, A. Héron ${ }^{\mathrm{b}}$, J.-C. Adam ${ }^{\mathrm{b}}$, A. \\ Miscampbell ${ }^{c}$, G. Thomas ${ }^{\mathrm{a}}$, R. Royle ${ }^{\mathrm{c}}$, O. Humphries ${ }^{\mathrm{c}}$, R.H.H. Ko ${ }^{\mathrm{a}}$, S. Le Moal ${ }^{\mathrm{a}}$, A. Tan ${ }^{\mathrm{a}}$, \\ J. Li ${ }^{\mathrm{a}}$, T.R. Preston ${ }^{\mathrm{c}}$, Q. van den Berg ${ }^{\mathrm{c}}$, M. Kasimc ${ }^{\mathrm{c}}$, B. Nagler ${ }^{\mathrm{d}}$, E.C. Galtier ${ }^{\mathrm{d}}$, \\ E. Cunningham ${ }^{\mathrm{d}}$, J.S. Wark ${ }^{\mathrm{c}}$, and S.M. Vinko ${ }^{\mathrm{c}}$ \\ ${ }^{a}$ Department of Physics, University of Toronto, 60 Saint George Street, Toronto, ON, \\ M5S 1A7, Canada \\ ${ }^{\mathrm{b}} \mathrm{CPHT}$, CNRS, Ecole Polytechnique, Institut Polytechnique de Paris, Palaiseau, France \\ ${ }^{c}$ Department of Physics, University of Oxford, Parks Rd, Oxford OX1 3PU, UK \\ ${ }^{\mathrm{d}}$ SLAC National Accelerator Laboratory, 2575 Sand Hill Road, Menlo Park, CA 94025, USA
}

\begin{abstract}
Conventional solid-density laser-plasma targets quickly ionize to make a plasma mirror, which largely reflects ultra-intense laser pulses. This Fresnel reflection at the plane boundary largely wastes our efforts at ultra-intense laser/solid interaction, and limits target heating to nonlinear generation of high-energy electrons which penetrate inward. One way around this dual problem is to create a material with an anisotropic dielectric function, for instance by nanostructuring a material in such a way that it cannot support the material responses which generate a specularly reflected beam. We present linear theory for metallic and plasma nanowires, particle-incell simulations of the interaction of ultra-intense femtosecond pulses with nickel nanowires, showing penetration of laser light far deeper than a nickel skin-depth, helping to uniformly heat near-solid material to conditions of high energy-densities, and XFEL experiments giving insight into their ionization and excitation.
\end{abstract}

\section{INTRODUCTION}

Since the 18th century, scientists have deliberately tried to increase the efficiency of light absorption. For example, the first reported experiment to build a solar oven was conducted by Nicholas de Saussure (1740-1799), ${ }^{1}$ and consisted of spaced glass blocks on top of a blackened surface enclosed by an insulated box. Adding a black coating on the glass surface, he was able to significantly increase the temperature inside. In modern times, it has been shown that the use of textured surfaces can permit a significant increase in absorption. ${ }^{2}$

In homogeneous matter optical absorption is macroscopically described by the complex-valued refractive index. Modifying the molecular properties of a material, such as the chemical or crystal structure, can engineer this index inside the bulk; the addition of antireflective multilayer coatings is a well-established technology for modifying net reflection at the interface by way of coherent interference at a series of constructed interfaces. Recently, nanostructured layers have been introduced to grade the refractive index from the first surface to the substrate, allowing very high absorptivity for a large range of incidence angles over the whole visible spectrum. ${ }^{3,4}$ In those devices, the first layers are composed of nanowires with different properties (composition, orientation and fill-factor), allowing a refractive index close to one at the first surface, and from there small step increases from vacuum to solid.

The goal of shaping a material, with nanometer-scale features, is to alter optical properties by changing the boundary conditions of the material on a wavelength or sub-wavelength scale. Structural scattering factors may be introduced (e.g., Mie scattering, ${ }^{5}$ etc.), and deliberate anisotropies may be designed into the material; the

Further author information: (Send correspondence to R.S.M.)

R.S.M.: email: marj@physics.utoronto.ca

G.K: current address Laser Impulse GmbH, Wiesenkamp 30, 24226 Heikendorf, Germany http://www.laserimpulse.com

L.L.: current address CEA-DAM-DIF, 91297 Arpajon, France

T.R.P.: current address European XFEL GmbH, Holzkoppel 4, 22869 Schenefeld, Germany 
average density of the material may be engineered to be different from the bulk, allowing new opportunities to direct or to control absorption. ${ }^{6}$ Individual nanowires (or nanorods) have been studied for their response to solar illumination, ${ }^{7}$ as have ensembles of nanowires as a nanomaterial. ${ }^{8-10}$

Laser-matter interaction in the ultra-intense $\left(>10^{18} \mathrm{Wcm}^{-2}\right)$ and ultrafast $(<100 \mathrm{fs})$ regime is almost universally characterized by low optical absorption, because in as little as tens of femtoseconds a metal-like, highly ionized, near-solid-density plasma is produced even from glass and often dielectrics. At the laser-plasma interface of a conventional flat solid target, the large and abrupt change in the index of refraction leads to large Fresnel reflectivity, and much-reduced coupling of laser energy into the target. Nanostructured targets afford the opportunity to modify these Fresnel coupling losses, greatly increasing absorption of the laser within the dense plasma, and producing significantly more-intense x-ray emission.

Metallic nanostructured targets composed of quasi-fractal porous aluminum or gold ('gold-black') targets, much like carbon candle-soot, and targets patterned with nanogrooves have been studied as high-absorption laser-plasma targets, especially with the aim of producing intense small-source-size picosecond-duration x-ray sources. ${ }^{11}$ Other sorts of nanostructured targets have since been reported by other groups; in particular, we first introduced nickel nanowires as laser-plasma targets, ${ }^{12}$ which from intense 1-ps pulses at $\lambda=1054 \mathrm{~nm}$ produce intense few-picosecond pulses of kiloelectronvolt x-rays. ${ }^{13}$ Subsequently, several studies have reported progress in using different kinds of nanostructured materials as experimental interaction-studies at progressively higher intensities and shorter pulse-durations. ${ }^{14-17}$ In recent years, nanowire materials used as laser-plasma targets have been shown to be able to produce exceptional conditions of high energy-densities, resulting in extraordinarily high ionization states and gigabar pressures, otherwise accessible only in laser-fusion compressed cores. ${ }^{18}$ Thus, nanowire targets open the study of exotic high-energy-density plasmas to faster development, by researchers using modest-sized laser systems.

Figure 1 shows a SEM image of a nickel-nanowire target of the type used in studies of laser-plasma interactions. ${ }^{13}$ This material consists of a surface of end-standing 10-200nm diameter metallic fibers, in a structure resembling velvet fabric (Fig. 1), which may be cheaply fabricated by electrochemical means from a range of different atomic elements from aluminum to platinum. ${ }^{12}$ Individual fibers are near-solid density, and the average

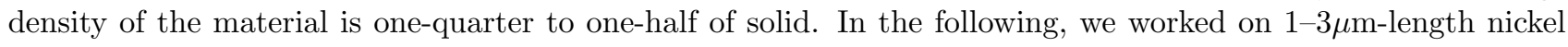
nanowires of $\sim 70 \mathrm{~nm}$ diameter and $\sim 70 \mathrm{~nm}$ spacing.

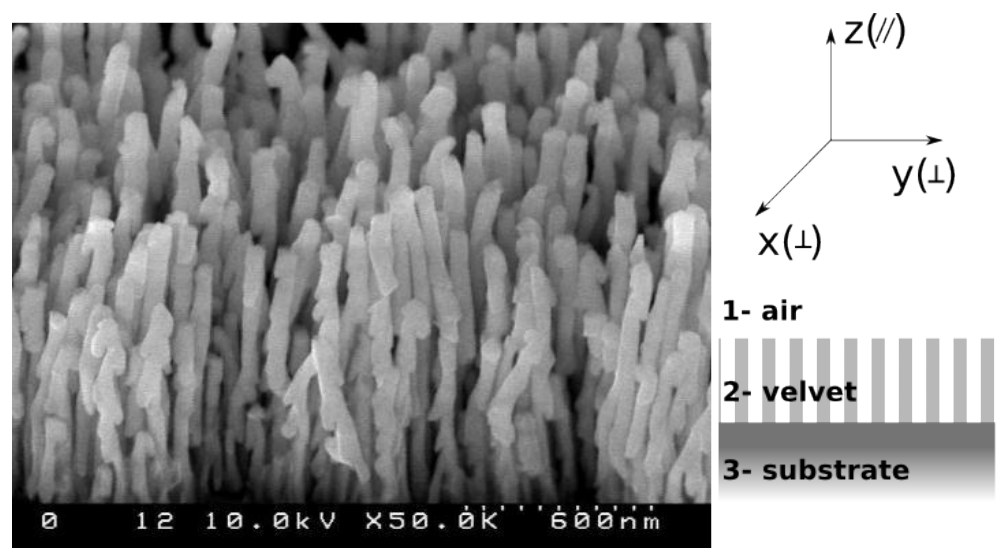

Figure 1. SEM picture of nickel-nanowire surface. $1 \mu \mathrm{m}$ length nanowires here have $\sim 70 \mathrm{~nm}$ diameter, and $\sim 70 \mathrm{~nm}$ spacing, with an effective density $\sim 1 / 4$ solid.

In this paper we describe the modelling of small-signal absorption in such metallic-nanowire targets, using an effective-dielectric approach, to better understand the physical optics issues of linear absorption at low intensities, and as a first step towards understanding the behaviour of these materials used as laser-plasma targets. Predictions of this theory are then compared to measurements of low-intensity absorption in Ni nanowires. For high-intensity applications, we describe the results of particle-in-cell simulations in two dimensions. Our experiments irradiating these targets at relativistic intensities up to a few times $10^{19} \mathrm{Wcm}^{-2}$ are described, and finally 
we make specific observations about the importance of extremely high temporal contrast when irradiating these nanostructured targets.

\section{EFFECTIVE DIELECTRIC CALCULATIONS}

To describe propagation in nanostructured targets, we need to consider the induced polarization in the inhomogeneous and anisotropic structure, and the magnetization field if is significant. For many materials, most being non-magnetic, we need only consider the inhomogeneous and anisotropic polarization density. In our case, the nickel nanowires are magnetic, but at the frequency of visible light the magnetic permeability is negligibly low. ${ }^{19}$

The familiar dielectric function $\epsilon(\omega)$ of a homogeneous material may be recast for nanostructured materials as an effective dielectric function which is applicable macroscopically. ${ }^{20}$ In that case, the effective dielectric function is also a function of the material geometry on the nanoscale. Starting with a single wire (cylindrical geometry) where the diameter is much smaller than the wavelength, the total electric field $\vec{E}$ within a wire comprises both applied field and polarization response, and is well-represented by the formula: ${ }^{5}$

$$
\begin{aligned}
\vec{E} & =\vec{E}_{0}-L(4 \pi \vec{p}) \\
& =\vec{E}_{0}-L\left(\epsilon_{B}-1\right) \vec{E}
\end{aligned}
$$

where $\epsilon_{B}$ is the dielectric function of the bulk material, $\vec{E}_{0}$ is the applied field, $\vec{p}$ is the induced polarizationdensity in a single wire, and $L$, the form factor, is dependent on the applied field orientation: 0 or $1 / 2$ for $\vec{E}_{0}$ parallel or perpendicular to the wire, respectively. Note that $L=0$ indicates that the wire reacts as the bulk for applied fields parallel to the wire. This equation yields the macroscopic polarization for a single nanowire, which is different in longitudinal $(\|)$ and transverse $(\perp)$ directions:

$$
\begin{aligned}
& p_{\|}=\frac{\epsilon_{B}-1}{4 \pi} E_{0 \|} \\
& p_{\perp}=\frac{\epsilon_{B}-1}{2 \pi\left(\epsilon_{B}+1\right)} E_{0 \perp}
\end{aligned}
$$

While in the parallel-field case the single-nanowire polarization response is like the bulk metal, in the perpendicular case the individual rods respond as dielectrics, even for typical metals, since $\mathcal{R} e\left[\left(\epsilon_{B}-1\right) /\left(\epsilon_{B}+1\right)\right]>0$ (Eqn. 4).

To describe the 'velvet' nanowire macroscopic material, we define a fill factor $f$ (with $0<f<1$ ), representing the geometric density of wires. Spatial averaging is equivalent to multiplying the mesoscopic electric polarization by the fill-factor. In this way the total material polarization-response, $\vec{P}=f \vec{p}$, will give the effective dielectric function of the macroscopic medium:

$$
\begin{aligned}
\epsilon_{\|} & =1+f\left(\epsilon_{B}-1\right) \\
\epsilon_{\perp} & =1+\frac{2 f \frac{\epsilon_{B}-1}{\epsilon_{B}+1}}{1-f \frac{\epsilon_{B}-1}{\epsilon_{B}+1}}
\end{aligned}
$$

Note that for the limit-values of the fill-factor, the equations recover the vacuum and bulk-material cases appropriately: for $f=0$ we have $\epsilon_{\|}=\epsilon_{\perp}=1$, and for $f=1$ we have $\epsilon_{\|}=\epsilon_{\perp}=\epsilon_{B}$. The complex-valued $\epsilon_{B}$ for bulk nickel has been measured experimentally by different groups, with slightly different results. ${ }^{21-23}$ On the other hand, these differences do not impact the discussion and the results discussed in this paper; we used $\epsilon_{B}=-17.5+29 i$ for $\lambda=1.0 \mu \mathrm{m} .{ }^{21}$ For nickel-nanowire target, we present in Fig. 2 the results of these formulae for $\epsilon=(n+i \kappa)^{2}$, where $n$ is the real refractive index and $\kappa$ is the extinction index, as a function of the fill-factor $f$.

The real part of the dielectric constant illustrates the particular character of transmitted light: In the parallel case, i.e., polarization along the nanowires, it is everywhere less than unity, which is characteristic of a conductor; for $f>0.05$ (at this wavelength), it is negative - below the effective plasma frequency, this component is 


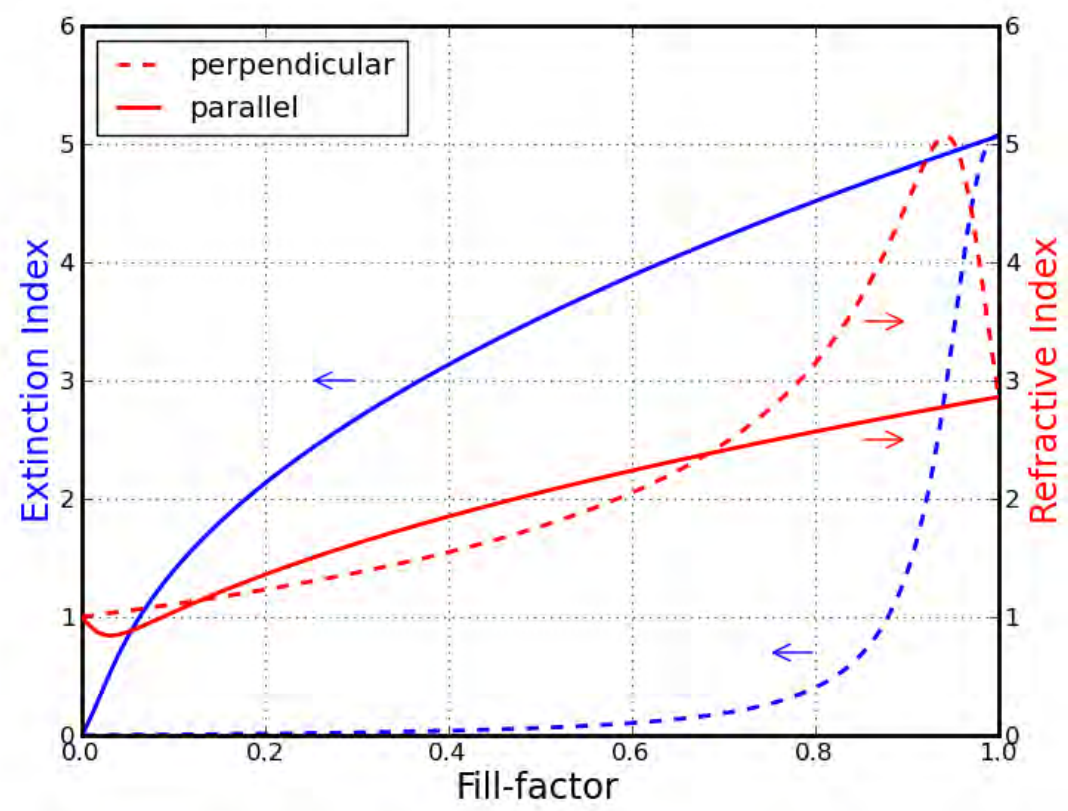

Figure 2. (Color online) The real refractive index (red) and the extinction index (blue) in Ni nanowire material, for parallel (solid lines) and perpendicular (dashed lines) applied electric fields $(\lambda=1 \mu \mathrm{m})$, as a function of the fill factor $0 \leq f \leq 1$.

evanescent. On the other hand, in the perpendicular case, $\mathcal{R} e\left[\epsilon_{\perp}\right]$ is everywhere greater than unity for fillfactors $f<0.95$, which is characteristic of a dielectric; and for $f<0.6$ absorption is relatively low. Coupling of light through the interface and into the nanowire material is much better than for bulk nickel, for both polarizations, but generally better for $E_{\perp}$ - and afterwards absorption is greater for $E_{\|}$. The skin depth is inversely proportional to the extinction index, and so the field will propagate much deeper for the perpendicular case, by about 2 orders of magnitude at 0.5 fill-factor. Therefore, EM waves incident at any angle quickly 'filter' to transmitted components which propagate in a direction along the nanowires.

In the following, we will consider the propagation of light into our oriented-nanowire targets, with the nanowires are perpendicular to the surface (see Fig. 1). The total dielectric of the target takes the form of a $3 \times 3$ tensor $\overline{\bar{\epsilon}}$ for which $\epsilon_{11}=\epsilon_{22}=\epsilon_{\perp}=$ and $\epsilon_{33}=\epsilon_{\|}$. This dielectric tensor formally connects the applied electric field $\vec{E}$ and the electric displacement field $\vec{D}=\overline{\bar{\epsilon}} \vec{E}$.

\section{PROPAGATION CALCULATIONS}

Using the standard self-consistent multilayer approach, ${ }^{24}$ we first find the Fresnel coefficients for field amplitudes in each polarization $\left(s\right.$ and $p$ ), at the interface between regions $i$ and $j^{25}$

We solve for three regions (see Fig. 1, (right)): a half-space of air (region 1), the oriented nanowire layer of thickness $d$ (region 2), and a half-space substrate of aluminum (region 3). Three steps are necessary: (i) solve Maxwell's equation for propagating waves within the nanowires, (ii) calculate the Fresnel coefficients at interfaces with our anisotropic medium, and (iii) calculate the reflected light from the whole structure using the transfer matrix formalism. ${ }^{26-29}$

With the Helmholtz equation and the dielectric function identified above, continuity requirements at an interface between regions $i$ and $j$ lead to the Fresnel coefficients for field amplitudes in each polarization $(s$ and $p)$ : 


$$
\begin{aligned}
& r_{i j}^{s}=\frac{w_{i}^{s}-w_{j}^{s}}{w_{i}^{s}+w_{j}^{s}} ; \quad r_{i j}^{p}=\frac{w_{i}^{p} \epsilon_{j}^{\perp}-w_{j}^{p} \epsilon_{i}^{\perp}}{w_{i}^{p} \epsilon_{j}^{\perp}+w_{j}^{p} \epsilon_{i}^{\perp}} \\
& t_{i j}^{s}=\frac{2 w_{i}^{s}}{w_{i}^{s}+w_{j}^{s}} ; \quad t_{i j}^{p}=\frac{2 n_{i} n_{j} w_{i}^{p}}{w_{i}^{p} \epsilon_{j}^{\perp}+w_{j}^{p} \epsilon_{i}^{\perp}}
\end{aligned}
$$

where $w^{s}=\sqrt{\tilde{\omega}^{2} \epsilon^{\perp}-k^{2}}, w^{p}=\sqrt{\tilde{\omega}^{2} \epsilon^{\perp}-\frac{\epsilon^{\perp}}{\epsilon} k^{2}}$, and $n_{i}=\sqrt{\epsilon_{i}^{\perp}}$, with $\tilde{\omega}=\omega / c$, and where $k$ is the component of the wave vector perpendicular to the nanowire (NB: here we refer parallel and perpendicular to the nanowire, as in, ${ }^{30}$ rather than to the interface planes of the layers, as $\left.i^{25}\right)$. Note that the $s$-polarization Fresnel coefficients only depend on the perpendicular-orientation dielectric function of the nanowires, whereas $p$-polarization involves the dielectric functions of both orientations.

With the assumption of a semi-infinite aluminum substrate (skin depth in aluminum is about $10 \mathrm{~nm}$ at 1 $\mu \mathrm{m}$ ), we obtain the net reflectivity of the 3-layer system (air, nanowires of thickness $d$, substrate):

$$
r_{n e t}^{s}=\frac{r_{12}^{s}+r_{23}^{s} e^{2 i w_{2} d}}{1-r_{21}^{s} r_{23}^{s} e^{2 i w_{2} d}}
$$

and similarly for $p$-polarization.

Fig. 3 depicts the modelled absorption $\left(A^{s, p}=1-\left|r_{\text {net }}^{s, p}\right|^{2}\right)$ for each polarization, as a function of fill-factor and angle of incidence, and for two different wavelengths: a) $\lambda=0.5 \mu \mathrm{m}, \mathrm{b}) 1 \mu \mathrm{m}$.

The thickness of the nanowire layer is $\sim 20 \mu \mathrm{m}$, i.e. much thicker than magnetic metals will allow, but which minimizes interference effects from reflections at the aluminum substrate underneath. As expected, the absorption is identical for $s$ - and $p$-polarizations in the limit of normal incidence. Similarly, the limiting case of fill-factor $f=1$ reproduces the bulk behaviour. The angle of incidence corresponding to maximum absorption of $p$-polarized light is traced (black line) for different fill-factors; for $f=1$ this angle corresponds to the principal angle of incidence for solid nickel (cf. Brewster's angle in dielectrics). The principal angle shifts to higher values, as the real part of the effective index of refraction increases with increasing fill-fraction; Fresnel losses are smallest at this value, leading to more-efficient in-coupling of light into the nanowire layer, after which the light is virtually completely absorbed within a micrometer or so.

Looking particularly at fill-fractions below 0.5 , the absorption is greater than $90 \%$ over a wide range of angles of incidence. It is worth noting again here that the material is composed of metallic nickel, which in bulk is highly light-reflecting (i.e. for a fill factor $f=1$ ) but which when nanostructured is highly transmissive at the surface.

At the very lowest values of fill-fraction $(f<0.1)$, Fig. 3 shows a sudden drop in absorption for all angles of incidence. Indeed, the Fresnel coefficients are a combination of both $\epsilon_{\perp}$ and $\epsilon_{\|}$, but as shown in Section 2 above, $\epsilon_{\|}$behaves like a dielectric with a refractive index below 1 . In this case, when the angle of incidence exceeds the critical angle $\left(\theta_{c}=\sin ^{-1}(1 / n)\right)$, we have the total internal reflection. For low values of $f$ the calculated absorption is a combination of a dielectric with $n>1\left(\epsilon_{\perp}\right.$ part $)$ and $n<1\left(\epsilon_{\|}\right.$part $)$, which explains the sudden drop in absorption.

The physical picture of optics in nanowire targets can be described heuristically the following way: a normal metal supports currents at the interface and material fields inside, driven by the incident electromagnetic wave; these in turn radiate to give the specularly reflected field. The same currents and material fields also radiate forward-going fields which destructively interfere with the forward-going incident field to produce attenuation. In a nanowire target, the anisotropic material does not support the same constitutive response, so the reflected field is weaker and the net forward-propagating field stronger: effectively it cannot support the same Fresnel reflection as does bulk metal. As can be inferred from Fig. 2, the material response to $p$-polarized light is largely along the length of the wires, for $f<0.8$. Consequently, the radiation pattern of each wire is much like that of a linear antenna, peaking laterally and sending very little in direction of the target normal - either specularly or forward-going. Thus light is transmitted well through the surface and into the highly absorbing metal. 
As the thickness of the nanowire layer is reduced, light will reflect from the aluminium substrate, and thinlayer interference will create more complex structures the absorption patterns. In the same scheme as the left plot, in Fig. 3, the right plot shows the absorption resulting from a much thinner nanowire layer $(1 \mu m)$.
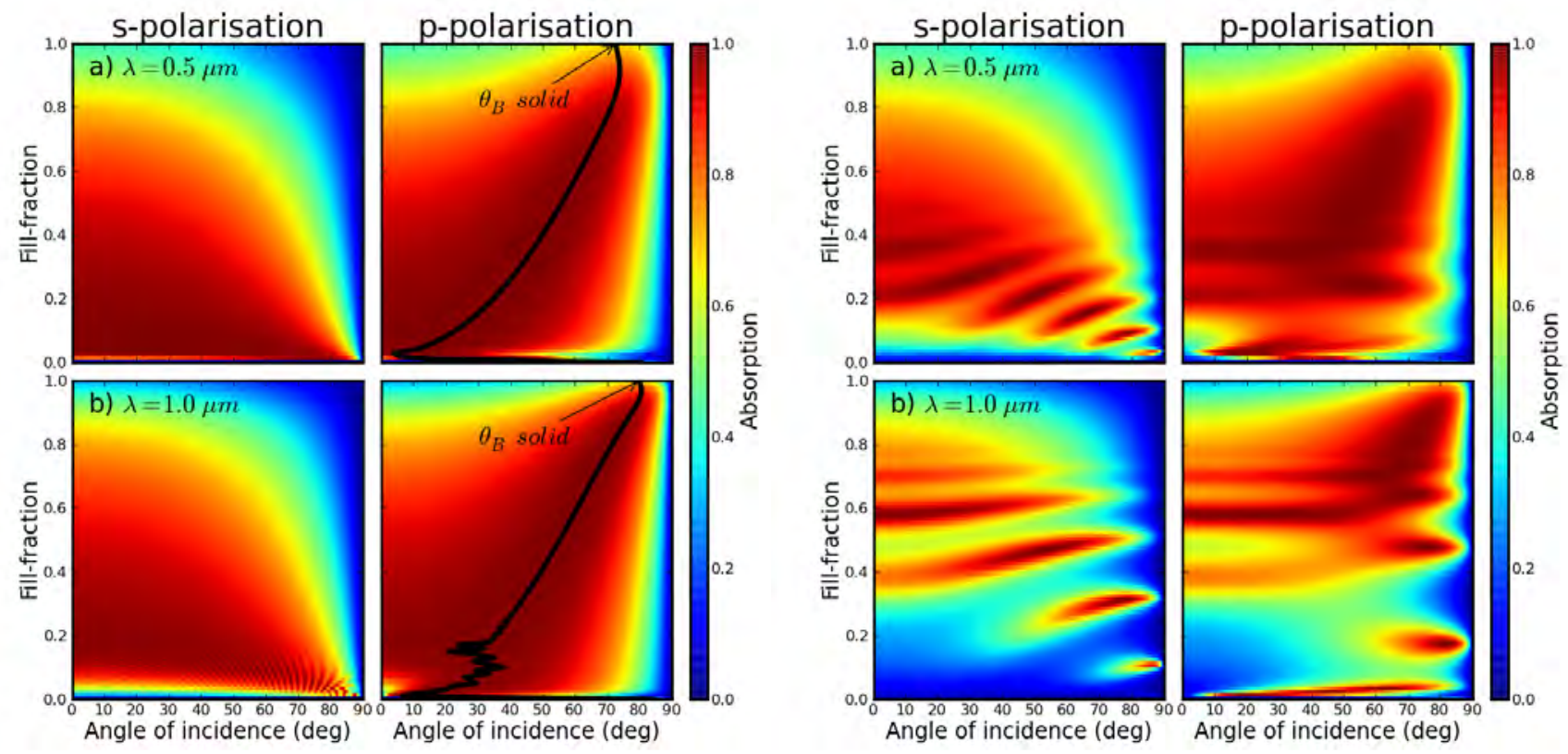

Figure 3. (Color online) Modelled absorption of $s$ - and $p$-polarized light incident on thick (Left: $\sim 20 \mu m$ ) and thin (Right: $1 \mu \mathrm{m}$ ) layers of nanowire, as a function of fill-fraction and angle of incidence, and for two different wavelengths: Row a) $\lambda=0.5 \mu \mathrm{m}$, Row b) $1 \mu \mathrm{m}$. Angles of maximum absorption are traced out in the $p$-polarization graphs (Left: black line) over different fill-factors; for $f=1$ this angle corresponds to the Brewster angle.

\section{EXPERIMENTAL COMPARISONS}

\subsection{Small Signal Measurements}

In order to validate our model-approximations, experimental measurements were performed for direct comparison. The source is a laser based on a diode-pumped neodymium-doped vanadate crystal, Q-switched and intracavity doubled, producing two wavelengths: $1.064 \mu \mathrm{m}$ and $0.5 \mu \mathrm{m}$. Filters were used to select wavelength, and a lens used to weakly focus the light onto the oriented-nanowire sample. The sample was held within an integrating (Ulbricht) sphere, and specular and scattered light collected by a photodiode. The incident light was also sampled from a beamsplitter, and measured at a second photodiode to give the reference intensity. This reference was normalized with no target in place, and then for samples under study the total reflectivity was taken as the ratio between the two signals obtained.

Fig. 4 compares the experimental (data points) and the modelled (solid lines) absorption, as a function of the angle of incidence for the two polarizations, and two wavelengths: a) $\lambda=0.5 \mu m$, and b) $\lambda=1.0 \mu m$. The modelling results are average over a small range of fill-factors $(f=0.5 \pm 0.05)$, to represent the small variation of inter-wire spacings in a real fabricated sample.

For the longer wavelength ( $1 \mu \mathrm{m}$, Fig. 4 (bottom)), theory and empirical result are in good agreement for both polarizations. The absorption for normal incidence is reproduced quite accurately; for the sample, though, both $s$ - and $p$-polarizations together show a small decrease in absorption immediately away from normal incidence, and this departure relative to modelling persists systematically for all angles. The maximum in absorption for $p$-polarization is clearly visible around $50^{\circ}$. At this angle, the absorption is much enhanced, compared to bulk 

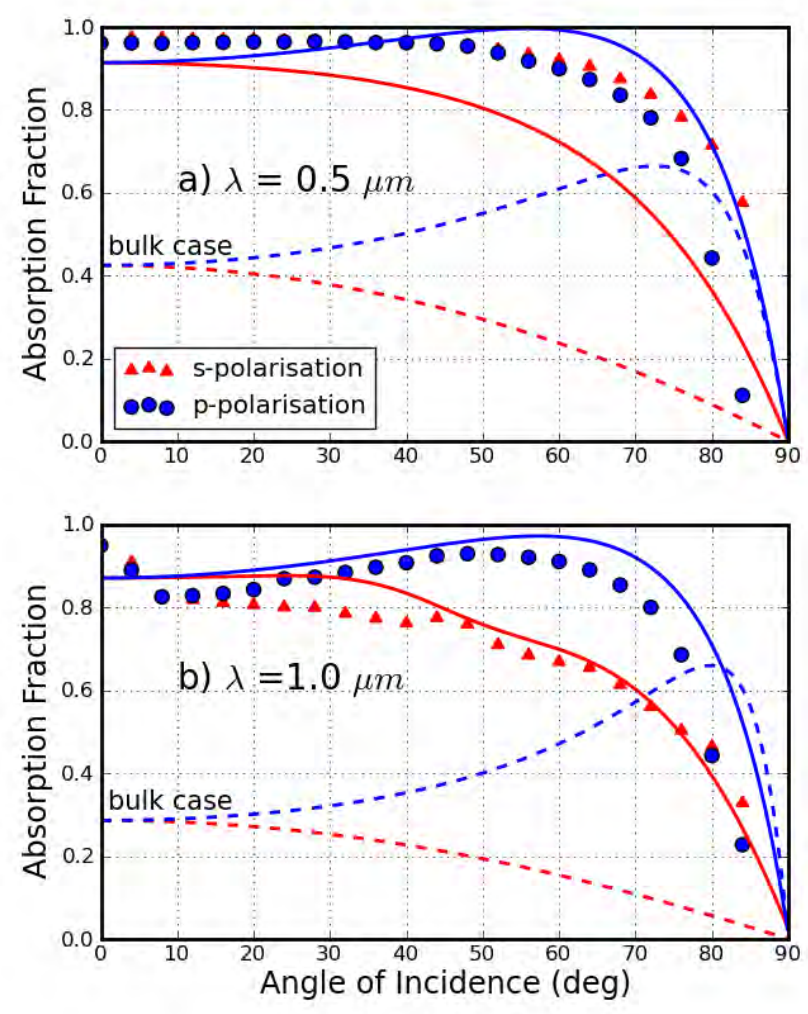

Figure 4. (Color online) Comparison of the experimental measurement (data points) and the theoretical calculation (solid lines) of the absorption as a function of the angle of incidence for the two polarizations (red is $s$-, blue is $p$-polarization), and different wavelengths: a) $\lambda=0.5 \mu \mathrm{m}$, and b) $\lambda=1.0 \mu \mathrm{m}$. For comparison, bulk absorption is shown (dashed lines).

nickel, reaching above 95\% (dashed lines in Fig. 4). Moreover, we measure comparably high absorption over a broad range of angles of incidence, between 0 to $70^{\circ}$.

At shorter wavelength $(0.5 \mu \mathrm{m}$, Fig. 4 (top) ) for the same sample, the agreement is not as good, but the model reproduces the high absorption for both polarizations. The experimental sample does not show significant differences between $s$ - and $p$-polarization until angles of incidence greater than $70^{\circ}$.

The principal assumptions of the model are: 1) that the wires are thin compared to a wavelength, 2) magnetic effects are unimportant, 3) that the wires are effectively infinitely long cylinders, in context of the geometrical effects in the form factors $L$ in equations (1) and (2) above, 4) that the fill-fraction $f$ accurately represents the distribution of wires (that local density-of-wires fluctuations are unimportant), 5) that the wires are oriented to be all parallel, and 6) that the bulk dielectric constant is valid to describe a single wire.

The first two assumptions are reasonably established, and are not expected to play a role in the agreement between modelling and measurements. The third assumption may play a role for thin layers, but likely the relevant comparison-length is not the wavelength of light, but the diameter of the wire. From scanning-electron micrographs of the targets, we estimate that the fill-fraction may show variation, but it is likely accounted for by averaging over a small range of fill-fractions, for wires of this diameter and spacing for which we do not expect very much impact from higher-order scattering: coupling between wires through their re-radiated field, which might reflect the local scale of inter-wire spacing.

However, scanning-electron micrographs of the targets do show significant distribution of angles of the nanowires, around the nominal orientation perpendicular to the substrate. This spread of angles can be larger for 
certain fabrication processes, and where the nanowire material is magnetized and the wires longer than roughly $1 \mu \mathrm{m}$, in which case pointed or ridged 'haystack' structures may result. Our modelling shows, though, that a range of angles is unlikely to have an important impact around most angles of incidence, since absorption depends only weakly on angle, except for near-grazing incidence.

\subsection{High Intensity Interaction}

Since their anisotropic dielectric function means that nanowire targets are effectively unable to support the material fields which are responsible for specular reflection, nanowire targets essentially do not have a Fresnel reflectivity, at least at modest intensities of light. This material has great potential for making much more efficient use of intense ultrafast laser pulses, since reflectivity can in principle be very low, but this depends on nanowires retaining their structure, during intense irradiation. Absorption may also be affected if the material's bulk index of refraction varies greatly as it is rapidly heated.

A rough prediction can be made for loss of nanostructure, based on the expectation that rapidly heated nanowires will explode, leading to void closure, and the subsequent homogenization of the nanostructured target. The speed at which the wires unload and expand into the vacuum between them is governed by the speed of the rarefaction wave into the nanowires. Nanowires of diameter $\sim 70 \mathrm{~nm}$ will be quickly heated by a cascade of laserdriven oscillating electrons released by ionization, and the rarefaction wave eroding them should be governed by the ion-acoustic speed of the heated plasma. For intensities $\mathrm{I} \sim 10^{17}-10^{19} \mathrm{Wcm}^{-2}$, this characteristic void-closure time is approximately $1 \mathrm{ps}$, down to roughly $100 \mathrm{fs}$.

\subsubsection{Particle-in-Cell Simulations}

Particle-in-cell simulations are particularly useful for studying the potential laser-plasma dynamics as ultrafast laser pulses propagate into the nanowire material, especially at relativistic intensities. The 2D PIC code EMI2D (Héron \& Adam) was used to assess the changing reflectivity of nanowires during irradiation. Fig. 5 compares

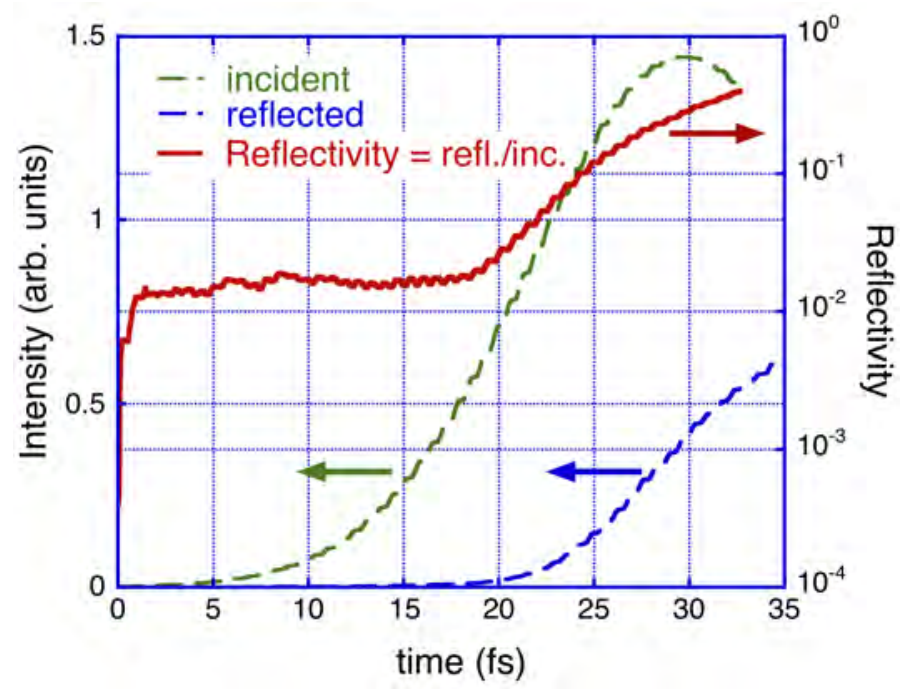

Figure 5. (Color online) 2-Dimensional PIC Code (EMI2D) simulation of time-dependent reflectivity of nanowire targets irradiated at $2.5 \times 10^{19} \mathrm{Wcm}^{-2}$ with $\lambda=400 \mathrm{~nm}, 20 \mathrm{fs}\left(a_{0}=1.7\right)$.

the instantaneous incident and reflected laser pulse intensity as determined at the simulation input-boundary, and corrected for transit times to and from the nanowire surface. From this, an instantaneous reflectivity was determined over $35 \mathrm{fs}$, from early in the leading edge of the laser pulse.

Corresponding values of absorption around $98 \%$ were preserved in the nanowire layer for about half the risetime of a $20 \mathrm{fs}$ pulse, but dropped below $80 \%$ by the pulse peak - still a factor of 2-3 improvement in absorption efficiency over that of a flat metal nickel target. The simulations show that electrons are drawn first from tips 
of nanowires as the laser pulse arrives, and the reflectivity this free-electron gas supports has a clear impact on the propagation of the laser pulse into the bulk of nanowires.

Insight into electron dynamics can be gained from the evolution of their density in phase-space. Fig. 6 compares phase space plots $\left(x, p_{y}\right)$ (left figure) and $\left(x, p_{x}\right)$ (right figure). The left figure shows the transverse oscillation of electrons in the $p$-polarized laser field as they are simultaneously driven away from the nanowire surface, toward the laser. The right figure shows that electrons are carried down the interstitial spaces between nanowires, with a wavenumber twice that of the laser, and presumably corresponding to the $J \times B$ force. These electrons are carried to the right boundary of the nanowires layer, where they set up a space-charge field which leads to strong fields and the reflection of subsequent electrons back through the nanowires without spatial density-structure.
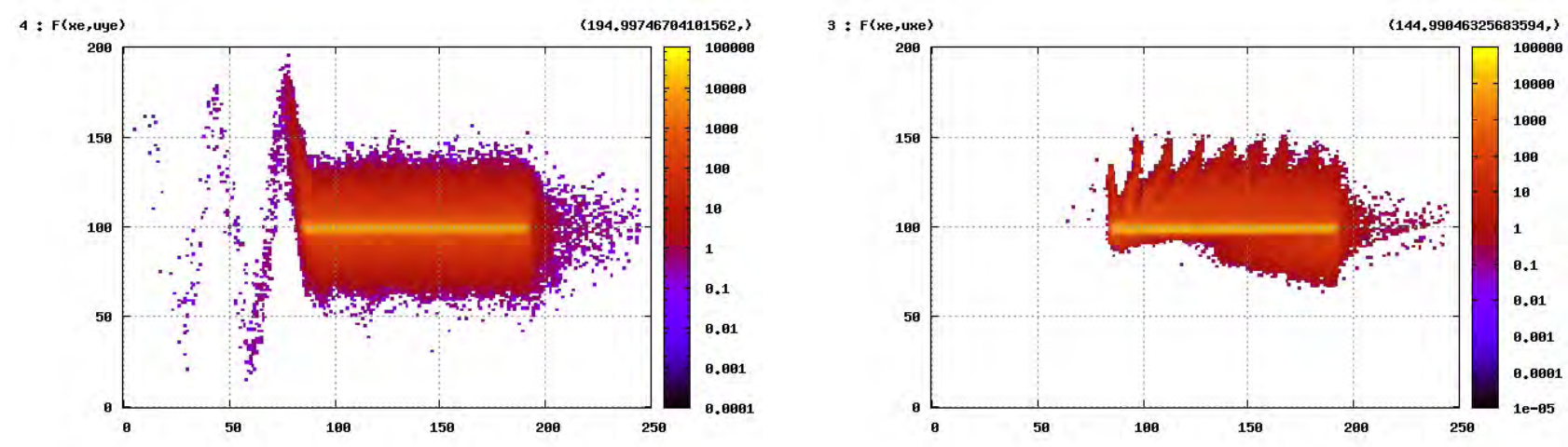

Figure 6. (Color online) 2-Dimensional PIC Code (EMI2D) simulation: Electron phase space plots for I $\sim 10^{19}$ Wcm ${ }^{-2}$. Left: $\left(x, p_{y}\right)$, at a time near the end of irradiation; Right: $\left(x, p_{x}\right)$, at a time showing the acceleration inward of strong currents of energetic electrons, their reflection from a free surface at right, and then their reflection as outward-going (negative momentum).

The electrostatic fields from electrons are imprinted on ions which are accelerated during and just after irradiation. Fig. 7 compares ion phase-space distributions for the conditions in Fig. 6 but at late times $\sim 1$ ps. The left-hand figure shows the phase space distribution of transversely moving ions $\left(x, p_{y}\right)$ at $1 \mathrm{ps.} \mathrm{In} \mathrm{the} \mathrm{body}$
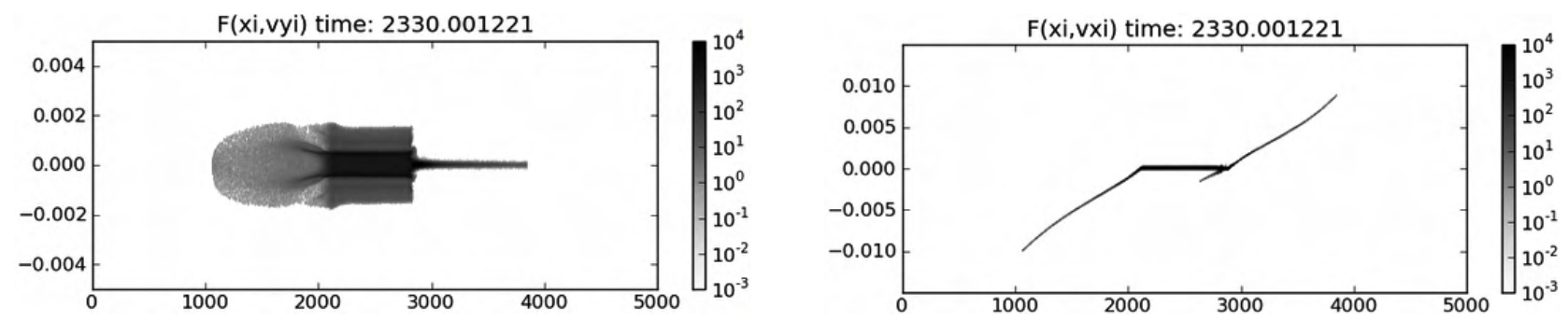

Figure 7. 2-D PIC Code (EMI2D) simulation: Ion phase space plots for I $\sim 10^{19} \mathrm{Wcm}^{-2}$. Left: $\left(x, p_{y}\right)$; Right: $\left(x, p_{x}\right)$.

of the material, this shows energetic ions from transversely expanding nanowires, due to the strong heating first of the electrons, and then a kind of Coulomb explosion of the non-neutral plasma formed. At the irradiated side of the nanowires, the ions expand energetically outwards and sideways. At the rear side of the free-standing zone of nanowires, the phase space distribution illustrates rear-side acceleration of ions in the space-charge field formed. The right-hand figure in Fig. 7 shows the longitudinal ion dynamics $\left(x, p_{x}\right)$, also at $1 \mathrm{ps,} \mathrm{long} \mathrm{after}$ irradiation. There's little longitudinal motion within the bulk of nanowires, but from the front and rear faces ions expand away from the nanowire layer with dispersion that shows their initial energy distribution. 


\subsubsection{High-Intensity Experiments}

Fig. 8 summarizes the time-integrated relative x-ray yields from Ni-nanowire targets and flat evaporated $\mathrm{Ni}$ targets, for $p$-polarized $\lambda=400 \mathrm{~nm}, 40$ fs duration laser pulses, as a function of incident laser energy in the range $I_{\text {laser }}=\operatorname{mid}-10^{16} \mathrm{Wcm}^{-2}$ to $10^{18} \mathrm{Wcm}^{-2}$. In Fig. 8 (left), the yield of XUV photons around $125 \mathrm{eV}$ is plotted, using a XUV diode coupled with a grazing-incidence gold mirror. In this soft range, nanowire targets produced yields similar to those from the flat $\mathrm{Ni}$ targets. The middle figure shows that for x-ray energies above $900 \mathrm{eV}$, the Ni-nanowire surfaces produce a yield increase of 10-40× over the flat metallic Ni target. The right figure shows that for x-ray energies above $1.5 \mathrm{keV}$, the nanowire produce about 10-20× the yield of flat metallic $\mathrm{Ni}$ targets. Over the laser-energy range studied, yields have a power-law dependence on laser energy $\left(E^{\alpha}\right.$ with $\alpha \sim 1.2-2$ ), and show no saturation.
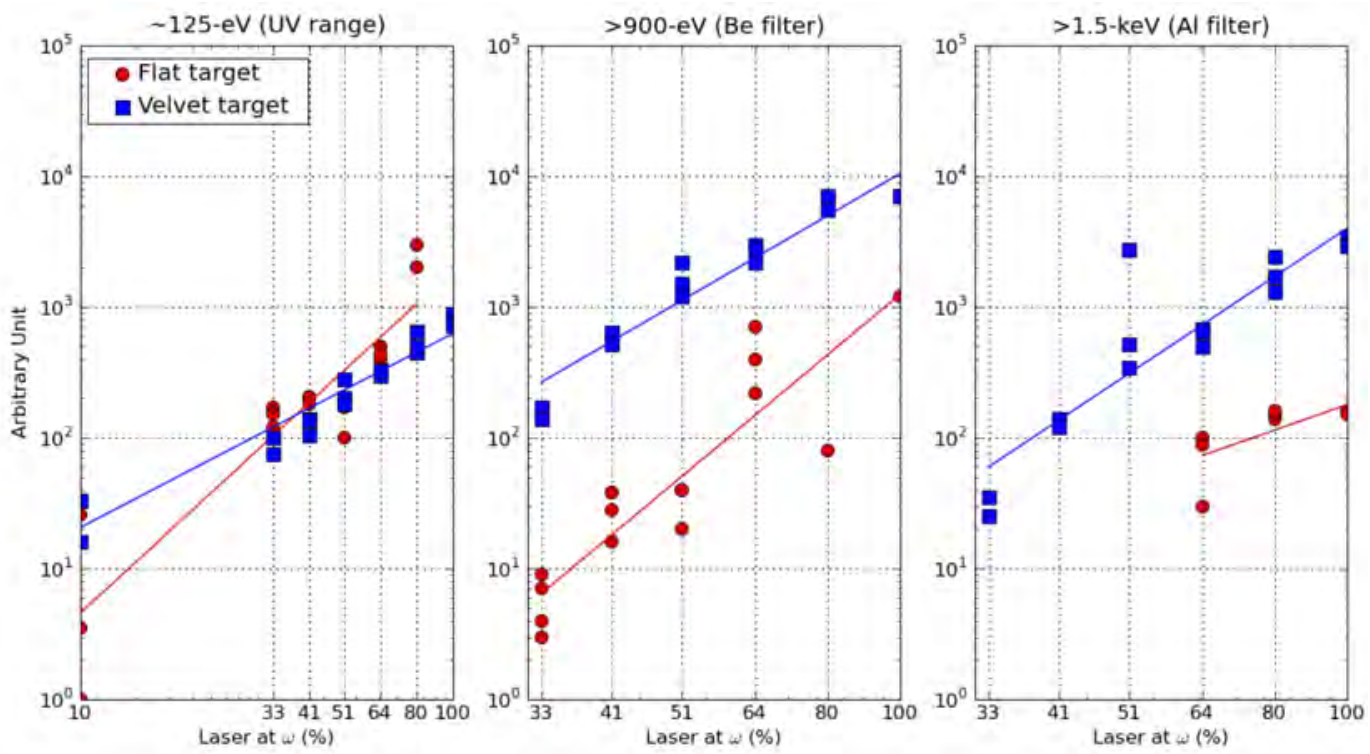

Figure 8. (Color online) XUV and soft x-ray conversion efficiency from flat Ni targets and Ni nanowire targets (ALLS $10 \mathrm{~Hz}$ laser system, $\left.\tau_{\text {laser }}=40 \mathrm{fs}, \lambda=400 \mathrm{~nm}\right), I_{\text {laser }}=$ mid- $10^{16} \mathrm{Wcm}^{-2}$ to $10^{18} \mathrm{Wcm}^{-2}$. Horizontal axes are $1 \omega$ $(800 \mathrm{~nm})$ power, before doubling.

\subsubsection{Importance of High Temporal Contrast}

Since nanowire targets combine high absorption efficiency with low mass structures and low thermal conductivity (along wire-length only), these targets require high temporal contrast - greater than is typically found even in very well engineered 10-TW-class (or higher) CPA lasers; frequency-doubling, or double plasma-mirror contrast enhancement is required. Very commonly, third-order autocorrelation is used to establish excellent contrast in the last few nanoseconds before arrival of the pulse, but accumulated fluences at nanosecond to microsecond times easily cause heating sufficient to destroy the nanostructures - particularly given the longer times for expansion before the arrival of the main pulse. It has been our nearly uniform experience of multiple top-tier laser user facilities that an undetected isolated prepulse at the contrast level of $10^{6}$ or larger exists at some time 5-200 ns in advance, or else that integrated amplifier ASE over a microsecond timescale before the pulse causes damage, despite the large solid-angle of emission.

Fig. 9 illustrates the issue in two cases. The left figure Fig. 9 shows the impact of oscillator pulses from the Toronto $\lambda=1054$-nm,1-ps CPA laser on a Ni-nanowire target. Gross damage from melting and ablation resulted from a fluence equivalent to a single $\sim 1 \mu \mathrm{J}$ pulse into a typical $6 \mu \mathrm{m}$-diameter experimental spot. The right-hand figure illustrates the damage caused to a Ni-nanowire target by Ti:sapphire-amplifier ASE from the $10 \mathrm{~Hz}$ ALLS laser (INRS-EMT). Between shots, our target was translated while being exposed to incidental ASE from the beamline amplifiers situated $\sim 10 \mathrm{~m}$ upstream, without any injected pulse. The nanowire structures 

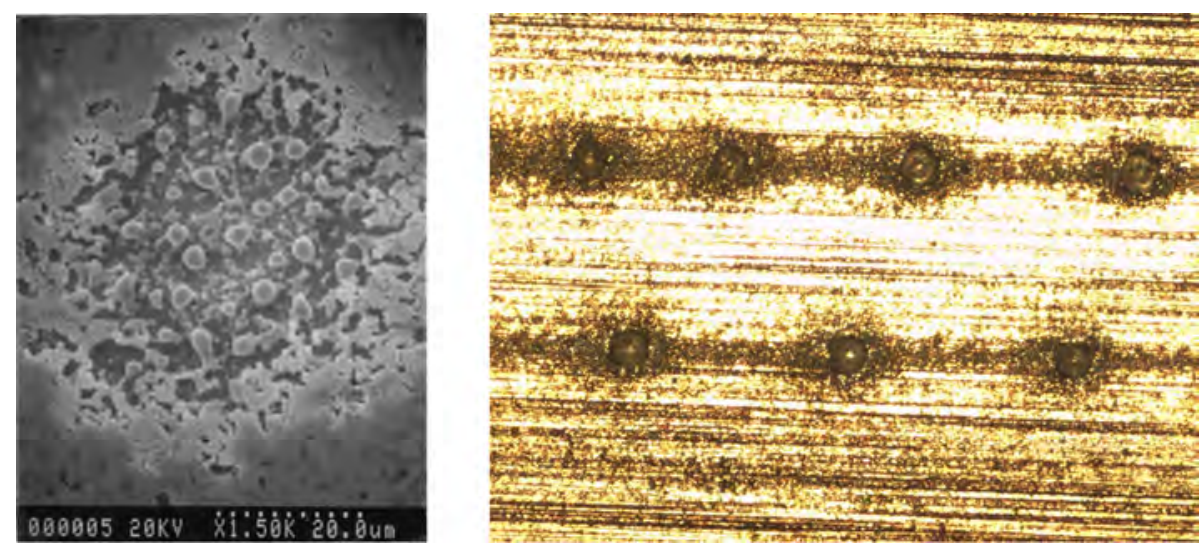

Figure 9. (Color online) Illustration of importance of high temporal contrast. Left: Impact of a single oscillator pulse from the Toronto $\lambda=1054-\mathrm{nm}$, 1-ps CPA laser on a Ni-nanowire target. Gross damage from melting and ablation resulted from fluence equivalent to a single $\sim 1 \mu \mathrm{J}$ pulse into $6 \mu \mathrm{m}$-diameter spot. Right: Impact of amplifier ASE (uninjected) from $10 \mathrm{~Hz}$ ALLS laser on Ni-nanowire targets. Between shots, the target was translated while exposed to beamline ASE from $10 \mathrm{~Hz}$ amplifiers situated $10 \mathrm{~m}$ upstream, without any injected pulse. The nanowire structures were destroyed before being shot, until a fast mechanical beam-shutter was deployed.

were destroyed before being shot, until we deployed a fast mechanical beam-shutter to isolate the target from the beamline until an injected-pulse shot was being taken.

Fig. 10 illustrates the impact, on these very susceptible targets, of standard temporal contrast from the same laser even with the beam-shutter in use. With $40 \mathrm{fs}$ pulses at $\lambda=800 \mathrm{~nm}$, and no double-plasma-mirror, the integrated fluence arriving unintentionally before the main pulse was sufficient to pre-form a plasma from Ni nanowires before arrival of the main pulse.

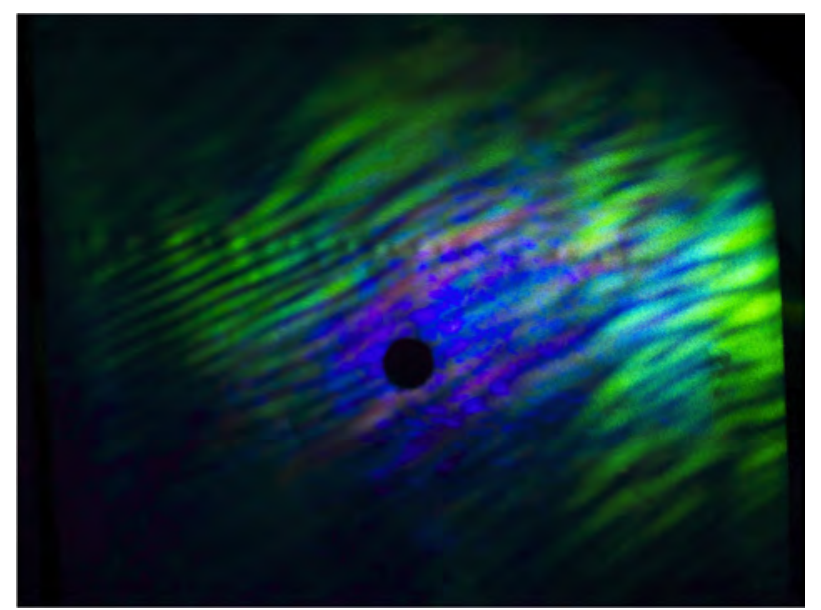

Figure 10. (Color online) True-colour reflectometry image from nanowires irradiated by the ALLS $10 \mathrm{~Hz}$ laser system (INRS-EMT) without double plasma-mirror, $40 \mathrm{fs}, \lambda=800 \mathrm{~nm}$. Integrated prepulse fluence was sufficient to preform a plasma from Ni nanowires before arrival of the main pulse. Note SHG (blue) and $\frac{3}{2}$ harmonic (green) from $\frac{1}{4}$-critical plasma. The dark central disk is a hole in the witness screen, passing the specular beam to a spectrograph.

The figure is a real-colour image of light generated from the target, within the forward-specular beam, as it is directed onto a flat-white witness screen and photographed. The pre-plasma is evidenced in the interference fringes seen in the far-field from the target, as fringes are affected by the thickness of the pre-plasma layer. SHG (blue) and $\frac{3}{2}$ harmonic (green) efficiencies are dependent on the scalelength of the pre-plasma at the half-critical and $\frac{1}{4}$-critical plasma surfaces. In effect, this imaged reflectometry arrangement makes a good and very simple test whereby the temporal contrast of the laser system can be tested in an integrated 'net result' way. 
The same method of imaged reflectometry shows great differences, when temporal contrast is high enough that the main pulse sees intact nanowires instead of pre-plasma. Fig. 11 illustrates the patterns of specularly reflected laser light from flat metallic Ni targets, and from Ni-nanowire targets, when irradiated at I $\sim 10^{19} \mathrm{Wcm}^{-2}$ using $40 \mathrm{fs}, 400 \mathrm{~mJ}$ frequency-doubled (400 nm) pulses (MEC optical laser, LCLS-SLAC). Flat Ni foils give a typical far-field pattern for the reflected laser pulse, while Ni nanowires greatly produce much less reflected light, imaged at the witness screen - the nanowires in this case kept their structural integrity well into irradiation by the main pulse.
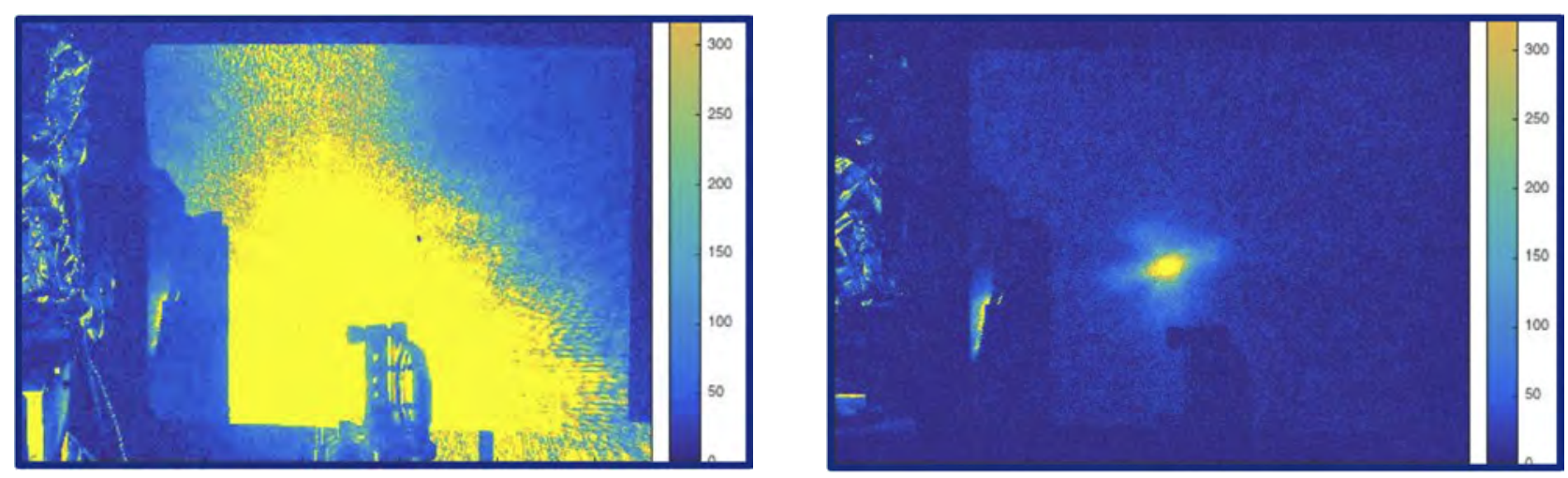

Figure 11. (Color online) Reflectometry images (false colour) of forward-reflected laser light from flat Ni target (left) and Ni nanowire target (right) $400 \mathrm{~mJ}$ optical laser pulse, $400 \mathrm{~nm}, 40 \mathrm{fs}, \mathrm{I} \sim 10^{19} \mathrm{Wcm}^{-2}$ (MEC optical laser, LCLS-SLAC). Left: Flat Ni foil gives typical specular-beam far-field reflection; Right: Ni nanowires give an order of magnitude reduced reflection - the nanowires keep their structural integrity well into irradiation by the main pulse. (To compare both with same intensity scale, the flat-target reflectometry image (left) is relatively saturated.)

Some of the arguments for using nanowire targets in intense laser-plasma interaction studies are captured in the PIC simulations of relativistic laser-particle dynamics (Fig. 6, Fig. 7). The observed increase in x-ray conversion efficiency for Ni nanowire targets over that for flat Ni (Fig. 8) likely results from increased optical absorption, together with increased total number of radiator-ions. The greater number of heated ions would be concomitant with the greater penetration-depth of an intense ultrafast laser pulse into nanowire materials compared to typical absorption skin-depths of a thin plasma from a solid target, as well as following from more-efficient direct deposition of energy, compared to typical secondary heating by radiation and hot-electron transport inward from a surface plasma.

Fig. 12 shows a first-principles calculation of conditions potentially achievable in Ni nanowire targets, starting from an ansat $z$ that a given laser-pulse energy is directly deposited into a thin cylindrical volume $14 \mu m$ diameter $\times 4 \mu \mathrm{m}$ deep lying directly under the focal spot footprint. This assumption leads to a presumed energy-density within the material, from which a self-consistent temperature for that energy-density can be found - for a given temperature, the atomic physics code $\mathrm{FLYCHK}^{31}$ was used to find ionization and excitation distributions, then to assess total energies of ionization and excitation for those temperature-dependent Ni-ion states. The net energy-density required to reach that electron temperature, and to pay the energetic costs for the concomitant ion states, was then compared to the energy-density available in the material from the laser pulse to determine the final temperature.

\section{CONCLUSIONS}

We have shown here the construction of an effective-medium model of an oriented nanowire material, considering the anisotropic polarization response and finding the material fields, building it into a thin-layer matrix formalism for self-consistent multi-surface reflections, to find the Fresnel reflection in the nanostructured material. We have predicted absorption of end-standing nanowires layers and its dependence on angle of incidence. Furthermore, we have measured and compared the absorption of such 'velvet' target for two wavelengths, as a function of the angle of incidence. We found a good agreement between the theoretical model and the experiment. 


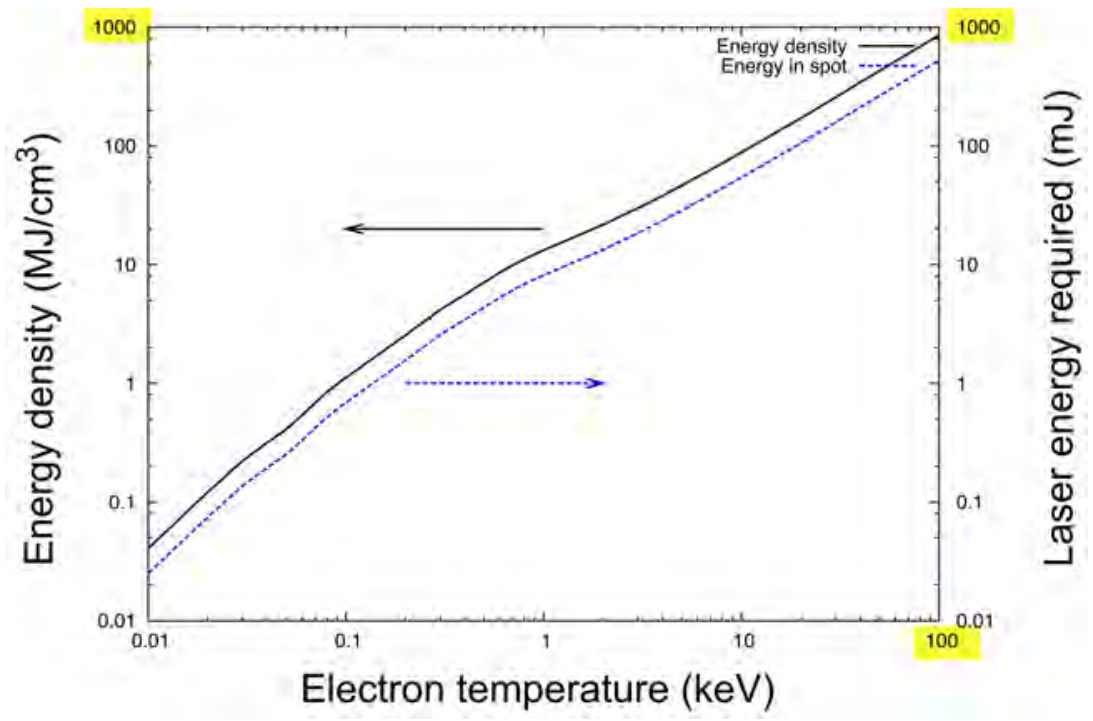

Figure 12. (Color online) Estimate from first principles of potential energy density, and plasma temperature found by assuming deposition of laser energy incident at the focal spot into a thin cylindrical volume $14 \mu \mathrm{m}$ diameter $\times 4 \mu \mathrm{m}$ deep. The absorbed energy leads to an energy density within the nanowire material. From this, the temperature is found self-consistently with the ionization modelling code FLYCHK.

We find that as a consequence of the reduction of Fresnel reflection, due to the nanostructured geometry, more light couples into the material, where it is absorbed but over a distance of $\sim 1 \mu \mathrm{m}$ - much larger than the skin-depth of bulk nickel - a length which is usually determined not by power dissipation in the material but by the scalelength of the evanescent field because the local plasma frequency exceeds the frequency of incident light. The collisionality and dissipation in the nickel is not really changed by nanostructuring, but because of Fresnel reductions more energy is coupled into the nickel nanowire structure, subsequently to be dissipated over a longer-than-bulk evanescent length. However, we note that under intense ultrashort-pulse laser irradiation, with the formation of warm or hot plasma from $\mathrm{Ni}$, the collisionality and dissipation in the warm plasma will change the modelling which here assumes a bulk-Ni dielectric function.

Since we first introduced nanowire materials as targets for ultrashort-pulse laser-plasma interaction studies ${ }^{13}$ there has been a strong interest in studying such nanostructured materials as high-intensity laser-matter interaction targets. We have shown results of 2D PIC simulations which highlight qualitatively distinct changes in the electron transport dynamics in oriented nanowire targets, as compared to flat solid targets. We also report experiments showing greatly enhanced conversion efficiencies into XUV and soft x-radiation.

These nanostructured targets are intrinsically much more sensitive to small levels of laser pre-pulse than are opaque solid targets, like flat metallic targets. Very few laser systems offer the intrinsic temporal intensitycontrast, or pre-pulse/main-pulse fluence contrast, required for intense laser-matter interaction studies with them, but enhancement by laser frequency-doubling or the use of double plasma-mirrors has here and elsewhere been established to be sufficient. We have shown that simply by imaging the specularly reflected beam, clear and qualitative differences in the image can serve as a straightforward 'qualification test' for the overall temporal intensity-contrast or energy-contrast of intense ultrashort-pulse laser systems.

\section{Acknowledgments}

R.S.M. acknowledges the support of the Natural Sciences and Engineering Research Council of Canada (NSERC). A.M., R.R., O.H., Q.vdB, M.K., J.S.W. and S.M.V. acknowledge support from the U.K. EPSRC under grant EP/P015794/1 and from the Royal Society. S.M.V. is a Royal Society University Research Fellow.

Use of the Linac Coherent Light Source (LCLS), SLAC National Accelerator Laboratory, is supported by the 
U.S. Department of Energy, Office of Science, Office of Basic Energy Sciences under Contract No. DE-AC02-

76SF00515. The MEC instrument is supported by the U.S. Department of Energy, Office of Science, Office of Fusion Energy Sciences under contract No. SF00515.

\section{REFERENCES}

[1] Meinel, A. B., "Applied solar energy: an introduction," (1976).

[2] Stephens, R. B. and Cody, G. D., "Optical reflectance and transmission of a textured surface," Thin Solid Films 45, 19-29 (Jan 1977).

[3] Xi, J.-Q., Schubert, M. F., Kim, J. K., Schubert, E. F., Chen, M., Lin, S.-Y., Liu, W., and Smart, J. A., "Optical thin-film materials with low refractive index for broadband elimination of Fresnel reflection," Nature Photonic 1, 176-179 (Jan 2007).

[4] Kuo, M., Poxson, D., Kim, Y., Mont, F., and Kim, J., "Realization of a near-perfect antireflection coating for silicon solar energy utilization," Optics Letters (Jan 2008).

[5] van de Hulst, H. C., "Light scattering by small particles," (Jan 1957).

[6] Fournier, K., Constantin, C., Poco, J., Miller, M., Back, C., Suter, L., Satcher, J., Davis, J., and Grun, J., "Efficient multi-kev x-ray sources from Ti-doped aerogel targets," Physical Review letters 92, 165005 (Jan 2004).

[7] Tian, B., Zheng, X., Kempa, T. J., Fang, Y., Yu, N., Yu, G., Huang, J., and Lieber, C. M., "Coaxial silicon nanowires as solar cells and nanoelectronic power sources," Nature 449, 885-U8 (Jan 2007).

[8] Ruda, H. and Shik, A., "Polarization-sensitive optical phenomena in semiconducting and metallic nanowires," Physical Review B 72, 115308 (Jan 2005).

[9] Ruda, H. E. and Shik, A., "Polarization-sensitive optical phenomena in thick semiconducting nanowires," Journal of Applied Physics 100, 024314 (Jan 2006).

[10] Muskens, O. L., Rivas, J. G., Algra, R. E., Bakkers, E. P. A. M., and Lagendijk, A., "Design of light scattering in nanowire materials for photovoltaic applications," Nano Lett 8, 2638-2642 (Jan 2008).

[11] Murnane, M. M., Kapteyn, H. C., Gordon, S. P., BOKOR, J., Glytsis, E. N., and Falcone, R. W., "Efficient coupling of high-intensity subpicosecond laser pulses into solids," Applied Physics Letters 62, 1068-1070 (Jan 1993).

[12] Al-Mawlawi, D., Liu, C., and Moskovits, M., "Nanowires formed in anodic oxide nanotemplates," J. Mater. Res (Jan 1994).

[13] Kulcsár, G., Al-Mawlawi, D., Budnik, F. W., Herman, P. R., Moskovits, M., Zhao, L., and Marjoribanks, R. S., "Intense picosecond x-ray pulses from laser plasmas by use of nanostructured "velvet" targets," Phys. Rev. Lett. (2000).

[14] Rajeev, P., Ayyub, P., Bagchi, S., and Kumar, G., "Nanostructures, local fields, and enhanced absorption in intense light-matter interaction," Optics Letters 29, 2662-2664 (Jan 2004).

[15] Rajeev, P. P., Kahaly, S., Bose, S., Kiran, P. P., Taneja, P., Ayyub, P., and Kumar, G. R., "Nanostructures and enhanced absorption in intense laser interaction with matter: effect of laser prepulses," ArXiv Physics e-prints (2005).

[16] Kahaly, S., Yadav, S. K., Wang, W. M., Sengupta, S., Sheng, Z. M., Das, A., Kaw, P. K., and Kumar, G. R., "Near-complete absorption of intense, ultrashort laser light by sub-lambda gratings," Physical Review letters 101, 145001 (Jan 2008).

[17] Wang, W. M., Sheng, Z. M., and Zhang, J., "A model for the efficient coupling between intense lasers and subwavelength grating targets," Phys Plasmas 15, 030702 (Jan 2008).

[18] Purvis, M. A., Shlyaptsev, V. N., Hollinger, R., Bargsten, C., Pukhov, A., Prieto, A., Wang, Y., Luther, B. M., Yin, L., Wang, S., and Rocca, J. J., "Relativistic plasma nanophotonics for ultrahigh energy density physics," Nature photonics 7, $796 \mathrm{EP}-$.

[19] Bruhat, G., "Optique," (Jan 1959).

[20] Kulcsár, G., Intense picosecond x-rays from structured targets, $\mathrm{PhD}$ thesis, University of Toronto, Toronto (1998). 
[21] Johnson, P. and Christy, R., "Optical-constants of transition-metals: Ti, V, Cr, Mn, Fe, Co, Ni, and Pd," Physical Review B 9, 5056-5070 (Jan 1974).

[22] Ordal, M., Bell, R., Alexander, R., Long, L., and Querry, M., "Optical properties of fourteen metals in the infrared and far infrared: $\mathrm{Al}, \mathrm{Co}, \mathrm{Cu}, \mathrm{Au}, \mathrm{Fe}, \mathrm{Pb}, \mathrm{Mo}, \mathrm{Ni}, \mathrm{Pd}, \mathrm{Pt}, \mathrm{Ag}, \mathrm{Ti}, \mathrm{V}$, and W," Applied Optics 24, 4493-4499 (Jan 1985).

[23] Palik, E. D., "Handbook of optical constants of solids, volume 1," 804 (1985).

[24] Gehr, R., Fischer, G., Boyd, R., and Sipe, J., "Nonlinear optical response of layered composite materials," Physical Review A 53, 2792-2798 (Dec 1996).

[25] Saarinen, J. J. and Sipe, J. E., "A Green function approach to surface optics in anisotropic media," J Mod Optic 55, 13-32 (Jan 2008).

[26] Sipe, J., "Bulk-selvedge coupling theory for the optical-properties of surfaces," Physical Review B 22, 15891599 (Jan 1980).

[27] Sipe, J., "The dipole antenna problem in surface physics - a new approach," Surf Sci 105, 489-504 (Jan 1981).

[28] Sipe, J., "New green-function formalism for surface optics," J Opt Soc Am B 4, 481-489 (Jan 1987).

[29] Knittl, Z., "Optics of thin film: An optical multilayer theory," (1976).

[30] Lugo, J. E., del Rio, J. A., and Tagüeña-Martínez, J., "Influence of surface coverage on the effective optical properties of porous silicon modeled as a si-wire array," J. Appl. Phys. 81, 1923 (Feb 1997).

[31] Chung, H. K., Chen, M. H., Morgan, W. L., density, Y. R. H. e., and 2005, "FLYCHK: Generalized population kinetics and spectral model for rapid spectroscopic analysis for all elements," Elsevier . 